'Departamento de Gastroenterología. Facultad de Medicina. Pontificia Universidad Católica de Chile. Santiago, Chile. Departamento de Hematología. Facultad de Medicina. Pontificia Universidad Católica de Chile. Santiago, Chile. ${ }^{3}$ Servicio de Medicina. Sección de Gastroenterología. Hospital del Salvador. Santiago Chile.

${ }^{4}$ Servicio de Medicina. Sección de Hematología. Hospital del Salvador. Santiago, Chile ${ }^{5}$ Departamento de Cirugía Digestiva. Facultad de Medicina. Pontificia Universidad Católica de Chile. Santiago, Chile.

${ }^{6}$ Programa de Trasplantes. Red de Salud UC-Christus. Santiago, Chile.

anterno de Medicina, Facultad de Medicina. Pontificia Universidad Católica de Chile. Santiago, Chile

${ }^{b}$ Enfermera Coordinadora. Programa de Trasplante Hepático. Facultad de Medicina. Pontificia Universidad Católica de Chile. Santiago, Chile.

Trabajo no recibió financiamiento Los autores declaran no tener conflictos de interés.

Recibido el 30 de julio de 2018, aceptado el 4 de marzo de 2019

Correspondencia a: Dr. Jorge Martínez C. Departamento de Cirugía Digestiva. Escuela de Medicina. Pontificia Universidad Católica de Chile.

Edificio Académico Facultad de Medicina. Diagonal Paraguay 362, Cuarto Piso. Santiago, Chile. jamartin@med.puc.c

\section{El trasplante de hígado es un procedimiento plausible, efectivo $y$ seguro en pacientes con hemofilia. Caso clínico}

\author{
CARLOS BENÍTEZ ${ }^{1,6}$, PAMELA ZÚÑIGA², FELIPE KRAMERª, \\ XIMENA VALLADARES ${ }^{4}$, PATRICIO ROJAS ${ }^{2}$, \\ EDUARDO PIMENTEL ${ }^{\mathrm{a}}$, FERNANDO GÓMEZ ${ }^{3}$, \\ ALEJANDRA CANCINO ${ }^{6, b}$, EDUARDO BRICEÑO ${ }^{5,6}$, \\ JUAN FRANCISCO GUERRA ${ }^{5,6}$, JORGE MARTÍNEZ ${ }^{5,6}$
}

\section{Liver transplantation in hemophilia A. Report of one case}

Due to blood derivative requirements, many patients with hemophilia were exposed to Hepatitis $C$ virus infection $(H C V)$ before the availability of HCV testing. We report a 46-year-old male with Hemophilia A with a hepatitis virus $C$ infection since 2004 causing a cirrhosis. Due to a hepatopulmonary syndrome, he received a liver allograph using a factor VIII replacement protocol, after eradicating the virus C. He had a good postoperative evolution, and no more factor VIII was required after transplantation until his last assessment.

(Rev Med Chile 2019; 147: 378-383)

Key words: Deficiency, Factor VIII; Hemophilia A; Liver Transplantation; Therapeutics, Liver Cirrhosis. $\tau$ a hemofilia se clasifica según el factor de coagulación deficiente, VIII para tipo A y factor IX (F.IX) para tipo B, y, según la actividad residual del factor, como grave (menos de $1 \%$ de actividad), moderada ( 1 a $5 \%$ ) y leve $(5 \text { a } 35 \%)^{1,2}$.

El factor VIII (F.VIII) de la coagulación es una proteína que se produce tanto en las células sinusoidales hepáticas como en células endoteliales ${ }^{3}$. Esta proteína circula en la sangre unida al factor von Willebrand y su principal función es ser un cofactor del F.IX (complejo tenasa) en la activación del factor $\mathrm{X}$, participando así en la fase de amplificación de la coagulación ${ }^{4}$.

La hemofilia se caracteriza por episodios de sangrados espontáneos o desproporcionados a la noxa; estos sangrados pueden ser graves e incluso fatales.
El tratamiento de la hemofilia se basa en la administración del factor deficiente (F-VIII-F.IX) ${ }^{5}$. En la década 1980-89 y principios de la década 1990-99, muchos pacientes con hemofilia se contagiaron del virus de inmunodeficiencia humana (VIH), virus de la hepatitis B (VHB) y virus de la hepatitis $\mathrm{C}$ (VHC), a través de concentrados de derivados sanguíneos liofilizados. En Chile el impacto fue menor, al no contar con el acceso a estos concentrados de F.VIII o F.IX. Sin embargo, una gran cantidad de pacientes con hemofilia fue expuesto a hemoderivados de donantes de sangre portadores de estas enfermedades ${ }^{6}$.

La hemofilia se incorporó a las Garantías Explícitas de Salud (GES) en el año 2006, principalmente para dar acceso a las personas con hemofilia al F-VIII o F-IX liofilizados con doble inactivación viral y buenos índices de bioseguridad. Esto signi- 
ficó un avance en la prevención de nuevos casos de hepatitis $\mathrm{C}$ adquiridos por transfusiones.

Actualmente, el trasplante hepático $(\mathrm{TH})$ es la mejor alternativa terapéutica para la insuficiencia hepática grave y las complicaciones de la cirrosis, tales como aquellas derivadas de la hipertensión portal o el hepatocarcinoma ${ }^{7,8}$. Este procedimiento representa un desafío mayor cuando la indicación del trasplante está en el contexto de un paciente con coagulopatía congénita ${ }^{9}$, dificultándose aun más de lo habitual la evaluación de la hemostasia en estos pacientes ${ }^{10}$.

Presentamos el caso del primer trasplante hepático $(\mathrm{TH})$ en Chile en un paciente con hemofilia y cirrosis secundaria a infección por VHC.

\section{Caso clínico}

Varón de 46 años con hemofilia A grave diagnosticada a los 2 años de vida tratado con múltiples transfusiones de crioprecipitados. A raíz de una artropatía hemofílica, se efectuó reemplazo articular de ambas rodillas en el año 2000. Se diagnosticó una hepatitis por VHC genotipo 1B en 2004, realizándose tratamiento con peginterferón/ribavirina, con nula respuesta. Evolucionó con una cirrosis hepática, hipertensión portal y várices esofágicas que presentaron sangrado grave en dos oportunidades. Esto determinó el inicio de tratamiento profiláctico con F.VIII liofilizado, 1.000 unidades tres veces por semana.

Durante 2015 presentó disnea progresiva, diagnosticándose un síndrome hepatopulmonar (SHP) con disminución paulatina de la $\mathrm{PaO}_{2}$, llegando a los $51 \mathrm{mmHg}$ con $\mathrm{FiO}_{2}$ ambiental. Se indicó oxigenoterapia domiciliaria y nos fue derivado para considerar un TH. Un comité multidisciplinario, que incluyó un hematólogo especialista en hemostasia, lo evaluó y enlistó para trasplante, con un score de MELD de 17 y un puntaje de Child-Pugh de 8 puntos.

En su estudio pretrasplante se confirmó serología positiva para VHC, enfermedad de Chagas, virus herpes simple 1 y 2, Epstein Barr, varicela zóster y citomegalovirus. Tenía serología negativa para VHB, indicándose inmunización.

Durante la espera del procedimiento, se inició esquema anti-VHC con sofosbuvir/ledipasvir/ ribavirina por 12 semanas. Mediante reacción en cadena de la polimerasa (PCR) a los 3 meses, se constató ausencia de infección por VHC. Adicionalmente, se indicó nifurotimox para el tratamiento de la enfermedad de Chagas con controles posteriores negativos. Se cuantificó inhibidores de F.VIII, que resultaron negativos y se diseñó un protocolo para manejo intra y postoperatorio.

Tras 11 meses alistado y con un MELD operacional de 22, se realizó un trasplante hepático utilizando un injerto de donante fallecido bajo protocolo de reemplazo de F.VIII: se administró una primera dosis en bolo para alcanzar un nivel de F.VIII en sangre entre 80 y $100 \%$ y posteriormente continuó con infusión continua para mantener estos mismos niveles o un tiempo R normal en el tromboelastograma (TEG) ${ }^{11}$ (Tabla 1). El trasplante se realizó con técnica de "Piggy-Back modificada" 12,13 y solo requirió transfusión de 3 unidades de glóbulos rojos de banco.

Se indicó esquema inmunosupresor utilizando tacrolimus, micofenolato y esteroides. Evolucionó satisfactoriamente con discreto aumento de aminotransferasas hasta AST (GOT): $448 \mathrm{UI} / \mathrm{L}$ y ALT (GPT): $278 \mathrm{UI} / \mathrm{L}$, con posterior tendencia a la normalización. Se extubó al segundo día postoperatorio.

La determinación seriada de F.VIII mostró una progresiva normalización (Figura 1), sin requerir del reemplazo de factores de la coagulación a partir del cuarto día postquirúrgico. Durante el postoperatorio presentó una sepsis de foco pulmonar e insuficiencia renal aguda, las cuales fueron tratadas acorde, evolucionando favorablemente. Previo al alta se controló con PCR para enfermedad de Chagas y VHC que fueron negativas.

En controles ambulatorios, mostró mejoría progresiva de su capacidad funcional respiratoria, sin complicaciones asociadas al trasplante o inmunosupresión, con $\mathrm{PaO}_{2}$ de $88,8 \mathrm{mmHg}$, sin necesidad de utilizar oxígeno domiciliario a partir del segundo mes postrasplante. No ha requerido de F.VIII de reemplazo hasta hoy (Figura 1).

\section{Discusión}

La infección por VHC es la principal causa de morbilidad y segunda causa de mortalidad en pacientes adultos con hemofilia expuestos a concentrados de factores de coagulación en décadas previas a 1990. Se describe internacionalmente que cerca de $90 \%$ de los pacientes expuestos, previo 
Tabla 1. Protocolo de reemplazo para trasplante hepático en hemofilia A

\begin{tabular}{|c|c|}
\hline \multirow[t]{5}{*}{ 1. Exámenes basales } & 1.1 Fibrinógeno: Valor esperado > 120 \\
\hline & 1.2 Recuento de plaquetas: $>50.000$ \\
\hline & 1.3 Tromboelastograma (TEG): Valor esperado R $>8$ (alterado por hemofilia) \\
\hline & 1.4 Factor VIII Basal $(\mathrm{F} \bullet \mathrm{VIII}):<1 \%$ \\
\hline & 1.4 Factor VIII Basal $(\mathrm{F} \bullet \mathrm{VIII}):<1 \%$ \\
\hline \multirow[t]{3}{*}{ 2. Preoperatorio } & 2.1 Tiempo 0 (previo postura catéteres): Administrar $\mathrm{F} \bullet \mathrm{VIII} 4.000 \mathrm{U}$ en bolo \\
\hline & 2.2 Tiempo 15 min (control F•VIII y TEG): TEG esperado: tiempo R se normaliza (4 a 8 min) \\
\hline & $\begin{array}{l}\text { Valor esperado } \mathrm{F} \bullet \mathrm{VIII} \text { : entre } 80-100 \% \\
\text { a) Si } \mathrm{F} \bullet \mathrm{VIII} \text { mayor } 80 \% \text { : Iniciar infusión } 2 \mathrm{u} / \mathrm{kg} / \mathrm{h} \text { según } 3.1 \\
\text { b) Si F•VIII entre } 50-79 \% \text {, administrar bolo adicional de } 1.000 \mathrm{U} \text { en } 10 \text { min e iniciar infusión en } \\
\text { pabellón según } 3.1\end{array}$ \\
\hline \multirow[t]{3}{*}{ 3. Intraoperatorio } & $\begin{array}{l}3.1 \text { Infusión continua de } \mathrm{F} \bullet \mathrm{VIII} 2 \mathrm{U} / \mathrm{Kg} / \mathrm{h} \text { (150 U/h). } \\
1.500 \mathrm{U} \text { de } \mathrm{F} \bullet \mathrm{VIII} \text { diluido en } 100 \mathrm{~mL} \text { de suero fisiológico a velocidad de infusión } 10 \mathrm{~mL} / \mathrm{h}\end{array}$ \\
\hline & 3.2 Tomar examen $\mathrm{F} \bullet \mathrm{VIII}$ y TEG cada $2 \mathrm{~h}$ desde 2.2 \\
\hline & 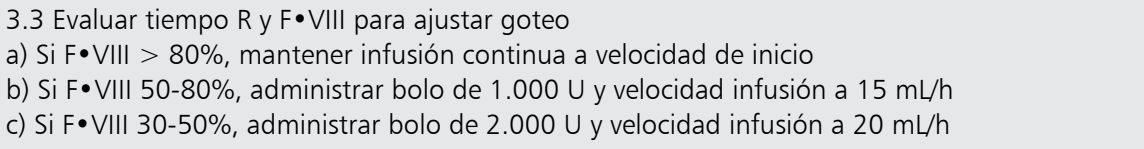 \\
\hline 4. Postoperatorio & $\begin{array}{l}\text { 4.1 Primeras } 48 \mathrm{~h} \text { mantener infusión continua de } \mathrm{F} \bullet \mathrm{VIII} \text { para mantener tiempo } \mathrm{R} \text { normal y } \mathrm{F} \bullet \mathrm{VIII} \\
\text { en } 80 \% \\
\text { Regular según } 3.3\end{array}$ \\
\hline
\end{tabular}

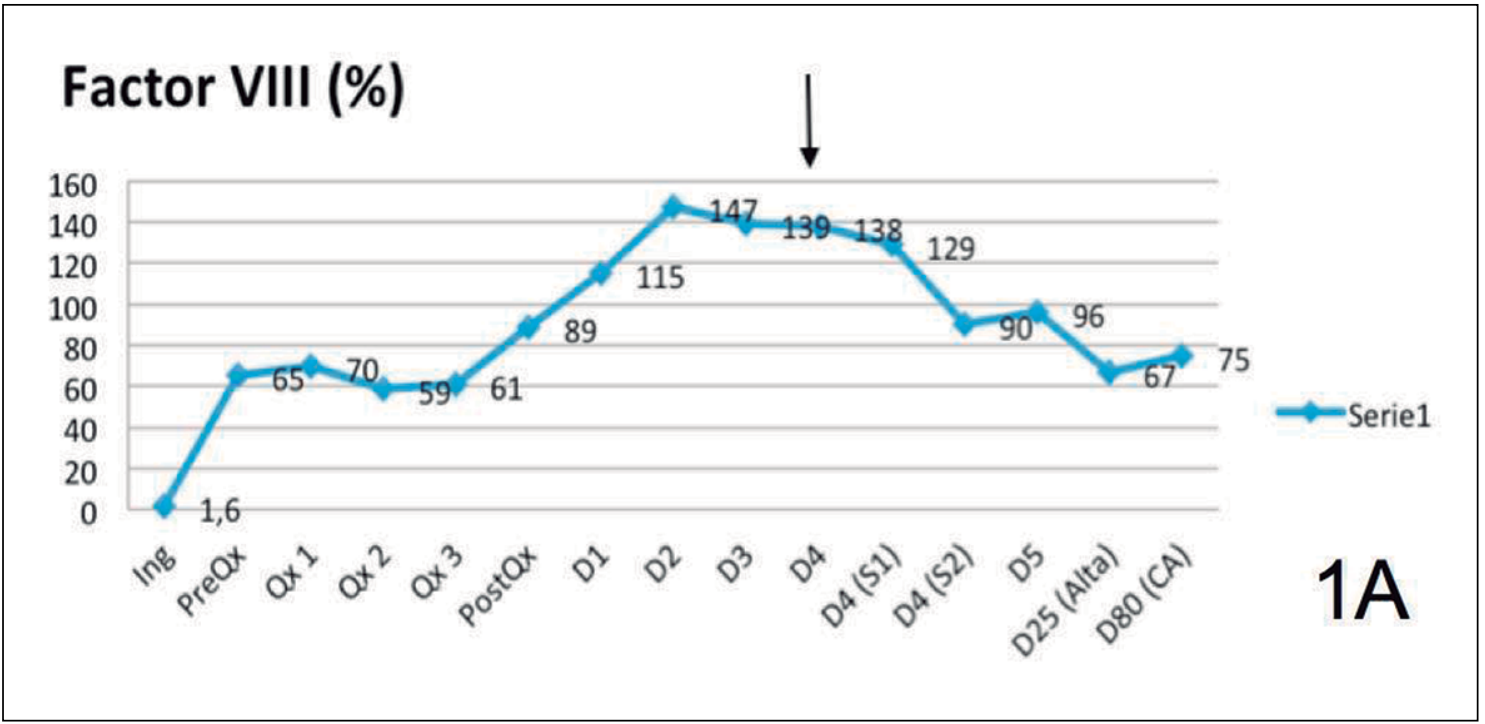

Figura 1A. Evolución de medición de FVIII (\%). Ing = Ingreso, PreQX = Pre quirúrgico, Qx = Durante la intervención quirúrgica, PostQx = Post operatorio, DX = Día X post operatorio, SX = Muestra X post suspensión de liofilizado, CA = Control ambulatorio. La flecha indica la suspensión de terapia de reemplazo de factores de la coagulación. 


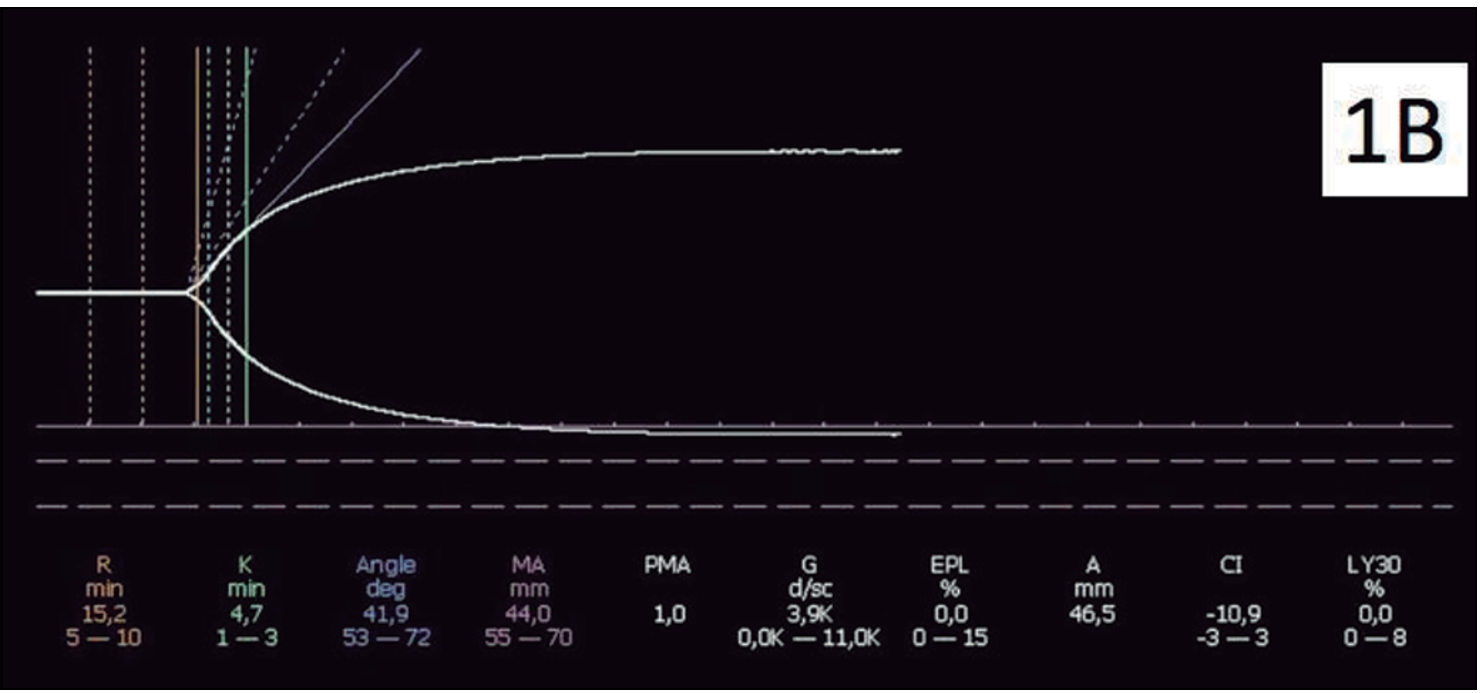

Figura 1B. TEG inicial con tiempo r prologado característico de déficit de Factor.

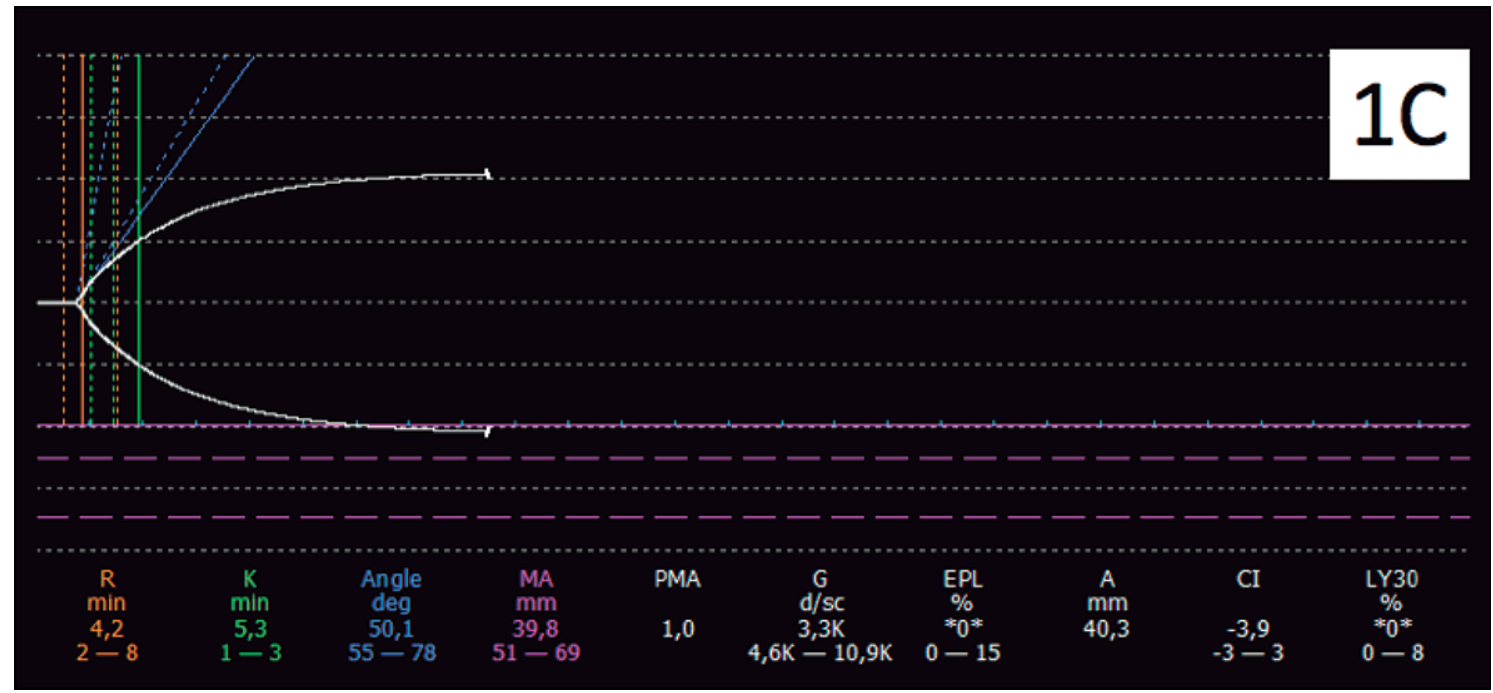

Figura 1C. TEG de seguimiento intrapabellón donde se observa mejoría del tiempo $r$ en relación a infusión de FVIII.

a la era del SIDA, fueron infectados por VHC y que aproximadamente $20 \%$ de los infectados por este virus evolucionó hacia cirrosis hepática ${ }^{14}$. La hepatitis crónica por VHC puede evolucionar en el curso de décadas generando fibrosis progresiva, lo que conlleva un elevado riesgo de cirrosis, hepatocarcinoma, hipertensión portal e insuficiencia hepática ${ }^{15}$. Aunque los antivirales de acción directa son capaces de erradicar la infección viral en una elevadísima proporción de pacientes y potencialmente revertir la fibrosis hepática, disminuir el riesgo de hepatocarcinoma y mejorar la función hepática, su disponibilidad en Chile es aún muy baja ${ }^{16}$.

El síndrome hepatopulmonar es una complicación de la hipertensión portal que produce hipoxemia progresiva y eventualmente grave, asociada a un aumento de la diferencia alveo- 
lo-arterial de oxígeno debido a la presencia de malformaciones vasculares que ocasionan cortocircuitos a nivel intrapulmonar ${ }^{17}$. El trasplante hepático se ha mostrado efectivo en estos pacientes con supervivencias de $70 \%$ y $64 \%$ a 1 y 2 años, respectivamente ${ }^{18}$.

En Chile, el tratamiento de la hemofilia con concentrados de F.VIII o F.IX con inactivación viral, incorporado al GES, es de alto costo. Nuestro paciente recibió tratamiento profiláctico desde su primer sangrado variceal, con 3.000 unidades de F.VIII a la semana, constantemente. Esto significó un importante incremento en el costo del tratamiento y deterioro importante de su calidad de vida.

La literatura ha descrito el rol del trasplante de hígado en pacientes con hemofilia ${ }^{19-22}$. Un trasplante exitoso determina, generalmente, una normalización de los niveles de F.VIII, como ocurrió en nuestro paciente.

Un equipo multidisciplinario que incluya un especialista en el tratamiento de coagulopatías y el acceso a terapia de reemplazo eficiente, disminuyen el riesgo quirúrgico. Un estudio reciente mostró que el riesgo del $\mathrm{TH}$ en pacientes con hemofilia, en un centro especializado, es similar a pacientes sin esta patología ${ }^{14}$. Así, la hemofilia no es una contraindicación para el TH. La mejoría en los niveles de F.VIII implica una mejoría en la calidad de vida y un ahorro significativo de recursos en el tratamiento de esta enfermedad. Específicamente en el caso de nuestro paciente, quien recibía al menos 12.000 unidades de F.VIII al mes, se ha ahorrado, en 18 meses 216.000 unidades de liofilizado.

Dados los buenos resultados del TH en nuestro medio, con supervivencias a 1,5 y 10 años de $82,3 \%, 70 \%$ y $64 \%$, respectivamente, y está financiado tanto en el sistema de salud público como por seguros privados, de forma que su costo no es un obstáculo al momento de ser indicado ${ }^{23}$, la disponibilidad actual de liofilizados y equipos con experiencia en el manejo de coagulopatias, la derivación de estos pacientes a un centro de trasplantes debería facilitarse. Si además consideramos que el TH ofrece la posibilidad de prescindir de tratamiento con concentrados de FVIII y que el tratamiento de erradicación del VHC está disponible, la indicación del trasplante en estos pacientes adquiere mayor relevancia. Esta herramienta terapéutica conlleva un mejor pronóstico global para el paciente y un menor gasto asociado al tratamiento crónico.

\section{Conclusión}

El trasplante hepático es una intervención posible en pacientes con hemofilia y hepatopatías crónicas, y como efecto adicional permite mejorar los niveles de F.VIII con un significativo impacto en la calidad de vida y ahorro sustancial de recursos.

\section{Referencias}

1. Hoyer L. Hemophilia A. N Engl J Med 1994; 330: 38-47.

2. Bolton-Maggs P, Pasi K. Haemophilias A and B. Lancet 2003; 361: 1801-9.

3. No H, Healey JF, Waller EK. Expression of factor VIII by murine liver sinusoidal endothelial cells. J Biol Chem 1999; 274: 19587-92.

4. Stel H, Van Der Kwast TG et Veerman E. Detection of factor VIII/coagulant antigen in human liver tissue. Nature 1983; 303: 530-2.

5. Key N, Negrier C. Coagulation factor concentrates: Past, present and future. Lancet 2007; 370: 439-48.

6. Donoso SM. Hemophilia in Chile, 1996-2006. Medwave 2013; 13: e5711.

7. Hepp J, Zapata R, Buckel E, Martínez J, Uribe M, Días J, et al. Trasplante hepático en Chile: Aspectos generales, indicaciones y contraindicaciones (Documento de consenso). Rev Med Chile 2008; 136: 793-804.

8. Neuberger J. An update on liver transplantation: A critical review. J Autoimmun 2016; 66: 51-9.

9. Forkin K, Colquhoun D, Nemergut E, Huffmyer J. The Coagulation Profile of End-Stage Liver Disease and Considerations for Intraoperative Management. Anesth Analg 2018; 126: 46-61.

10. Bezinover D, Dirkman D, Findlay J, Guta C, Hartmann M, Nicolau-Raducu R, et al. Perioperative Coagulation Management in Liver Transplant Recipients. Transplantation 2018; 102: 578-92.

11. Concha M, Mertz V, Muñoz G, Delfino A, Cortínez L, Montaña R, et al. Comparación preoperatoria entre pruebas de coagulación y tromboelastografía en pacientes con cirrosis hepática sometidos a trasplante hepático. Rev Med Chile 2018; 146: 277-81.

12. Belghiti J, Panis Y, Sauvanet A, Gayet B, Fékété F, et al. A new technique of side to side caval anastomosis during orthotopic hepatic transplatantion without inferior vena caval occlusion. Surg Gynecol Obstet 1992; 175: 270-2. 
13. Martínez J. Implantación con técnica de Piggy-Back y anastomosis cavo-cavo latero-lateral en trasplante hepático ortotópico: experiencia inicial en un centro universitario. Rev Chil Cir 2004; 3: 317-31.

14. Ragni MV, Ndimbe OK, Rice EO, Bontempo FA, Nedjar S. Presence of antibody to hepatitis $\mathrm{C}$ virus (HCV) in hemophilic men with HCV 'seroreversion'. Blood 1993; 82: 1010-5.

15. Westbrook RH, Dusheiko G. Natural history of hepatitis C. J Hepatol 2014; 61: 58-68.

16. European Association for the Study of the Liver. EASL Recommendations on Treatment of Hepatitis C 2018. J Hepatol 2018; 69 (2): 461-511.

17. Rodríguez-Roisin R, Krowka MJ. Hepatopulmonary syndrome a liver-induced lung vascular disorder. N Engl J Med 2008; 358 (22): 2378-87.

18. Raevens S, Rogiers X, Geerts A, Verhelst X, Samuel U, van Rosmalen $\mathrm{M}$, et al. Outcome of liver transplantation for hepatopulmonary syndrome: a Eurotransplant experience. Eur Respir J 2018. pii: 1801096.

19. Marion R, Delius R, Campbell D, Turcotte J. Othotopic liver transplantation totally corrects factor IX deficiency in hemophilia B. Surgery 1988; 104: 929-31.

20. Bontempo FA, Lewis JH, Gorenc TJ, Spero JA, Ragni $\mathrm{MV}$, Scott JP, et al. Liver transplantation in hemophilia A. Blood 1987; 69: 1721-4.

21. McCarthy M, Gane E, Pereira S, Tibbs C, Heaton N, Rela $\mathrm{M}$, et al. Liver transplantation for haemophiliacs with hepatitis C cirrhosis. Gut 1996; 39: 870-5.

22. Amaya O, Cuervo J, Raffan M, Manrique F, Martínez S. Orthotopic liver trasplant in a patient with Hemophilia A. Revista Colombiana de Anestesiología 2001; 39: 95 101.

23. Benítez C, Wolff R. Current Status and Future Challenges of Liver Transplantation Programs in Chile. Liver Transpl 2018; 24 (12): 1757-61. 